\title{
The Hanging Maneuver Facilitates Laparoscopic Right Hepatectomy
}

\author{
David A. Geller, MD
}

Division of Hepatobiliary and Pancreatic Surgery, Department of Surgery, UPMC Liver Cancer Center, University of Pittsburgh, Pittsburgh, PA

Laparoscopic hepatic resection is growing in number with more than 6,000 cases performed worldwide. ${ }^{1,2}$ Early series were predominantly nonanatomic liver resections, but greater experience has led to major anatomic hepatectomies being performed minimally invasively. ${ }^{3-5}$ Initial fears of adverse oncologic outcomes or tumor seeding have not been reported, and many studies have shown comparable 5-year overall survival in case-matched studies comparing laparoscopic to open hepatic resection for hepatocellular carcinoma or limited metastatic colorectal cancer. ${ }^{2}$ Benefits of minimally invasive hepatic resection include smaller incisions, less estimated blood loss and packed red blood cell transfusions, diminished narcotics, shorter length of stay, and decreased morbidity compared with open hepatic resection. ${ }^{2}$ Although two randomized clinical trials are ongoing in Europe comparing laparoscopic with open liver resection, none have been published to date, and it is important to note that selection bias may exist, even in well-matched series.

Formal anatomic laparoscopic right hepatic lobectomy remains a challenging operation because of technical factors that include inflow control, mobilizing a large right lobe liver (especially in an obese patient), dissection of the inferior vena cava (IVC) and control of the right hepatic vein, as well as parenchymal transection and control of back-bleeding from middle hepatic vein branches. Dagher

This is an invited editorial to the article available at doi: $10.1245 /$ s10434-014-3809-0.

\section{(C) Society of Surgical Oncology 2014}

First Received: 3 May 2014;

Published Online: 1 August 2014

D. A. Geller, MD

e-mail: gellerda@upmc.edu et al. ${ }^{3}$ reported a large series of 210 laparoscopic major lobectomies that included 136 formal right hepatic lobectomies; however, these cases were done at high-volume hepatopancreaticobiliary centers of excellence. A survey among 10 hepatopancreaticobiliary Centers in Europe reported 2,245 laparoscopic liver resections from 1996 to 2011, of which $348(15.5 \%)$ were laparoscopic right hepatectomies. ${ }^{4}$

The liver hanging maneuver (LHM) was originally described by Belghiti et al. ${ }^{6}$ in 2001 as a safe approach to facilitate open right hepatectomy via an anterior approach without liver mobilization. Two groups have reported their initial use of the LHM during laparoscopic hemihepatectomy. Nitta et al. ${ }^{7}$ reported using the LHM in 42 cases of laparoscopic-assisted major liver resection that included 14 laparoscopic right hepatectomies. They developed a forceps with a blunt and highly curved long tip to tunnel between the liver and the IVC. Troisi and Montalti ${ }^{8}$ used the Goldfinger dissector to perform a modified LHM in 13 right and 5 left hepatectomies by a pure laparoscopic approach. Neither group reported any bleeding complications from the blind passing of the instrument along the anterior surface of the IVC, which is the feared complication.

In the accompanying study, Dokmak et al. ${ }^{9}$ and colleagues provide a small series as well as a video of a laparoscopic right hepatectomy using an LHM. Specifically, they used a nasogastric tube to achieve a pure laparoscopic LHM tunneled from an up-to-down fashion starting at the right/middle hepatic vein space and emerging at the hilum without prior mobilization of the right liver. This is an elegant technique that is clearly demonstrated in the video on a 54-year-old woman with large telangiectatic adenomas. Potential advantages of the LHM (whether used during open or laparoscopic hepatic lobectomy) are elevation of the liver to guide the parenchymal 
transection, protection of the IVC, and decreased bleeding. Since 2011, the authors report 6 cases of laparoscopic right hepatectomy without LHM; however, 4 of those cases required hand assistance, and 2 cases were converted to open. Since adopting use of the laparoscopic LHM, the authors completed 5 laparoscopic right hepatectomies without hand assistance or conversion.

Although small numbers and not case-matched, the authors suggest that the LHM facilitated successful completion of the laparoscopic right hepatic lobectomy. A caveat is that the authors are a very experienced team and have been performing the LHM during open major hepatic resection for more than decade. It seems prudent that a hepatobiliary surgeon should be comfortable in performing the LHM during open hepatic resection before attempting it in a laparoscopic manner. However, the learning curve in performing open LHM before moving to laparoscopic has not been defined. The great concern in "blind passing" of an instrument in the tunnel along the anterior surface of the IVC to achieve the LHM is laceration of the IVC or avulsion of a short hepatic vein branch. None of the publications to date has adequately addressed how to handle such a crisis. It goes without saying that if bleeding is encountered, it will have to be addressed one way or another. Further, which instrument is used to traverse the tunnel and whether to go from up-to-down or down-to-up appear to be surgeon preference at this point and have not been studied in a systematic manner. Moreover, selection criteria as to which patients are eligible or ideal for laparoscopic major hepatectomy have not been established, and this will be one of the focus topics at the upcoming Second International Laparoscopic Liver Consensus Conference, to be held October 4-6, 2014, in Morioka, Japan. Further, whether the LHM can be used to facilitate a minimally invasive major hepatectomy in a patient with early Childs A cirrhosis has not been determined. The authors should be congratulated on presenting a technically superb operation and in demonstrating ingenuity in using a nasogastric tube to secure the LHM. Clearly, future investigation is needed to define the limits of this technique as well as patient selection criteria.

\section{REFERENCES}

1. Nguyen KT, Gamblin TC, Geller DA. World review of laparoscopic liver resection-2,804 patients. Ann Surg. 2009;250: 831-41.

2. Nguyen KT, Marsh JW, Tsung A, Steel JL, Gamblin TC, Geller DA. Comparative benefits of laparoscopic versus open hepatic resection: a critical appraisal. Arch Surg. 2011;146:348-56.

3. Dagher I, O'Rourke N, Geller DA, et al. Laparoscopic major hepatectomy: an evolution in standard of care. Ann Surg. 2009; 250:856-60.

4. Tzanis D, Shivathirthan N, Laurent A, et al. European experience of laparoscopic major hepatectomy. J Hepatobiliary Pancreat Sci. 2013;20:120-4.

5. Lin NC, Nitta H, Wakabayashi G. Laparoscopic major hepatectomy: a systematic literature review and comparison of 3 techniques. Ann Surg. 2013;257:205-13.

6. Belghiti J, Guevara OA, Noun R, et al. Liver hanging manoeuver: a safe approach to right hepatectomy without liver mobilization. $J$ Am Coll Surg. 2001;193:109-11.

7. Nitta H, Sasaki A, Fujita T, et al. Laparoscopy-assisted major liver resections employing a hanging technique: the original procedure. Ann Surg. 2010;251:450-3.

8. Troisi RI, Montalti R. Modified hanging maneuver using the goldfinger dissector in laparoscopic right and left hepatectomy. Dig Surg. 2012;29:463-7.

9. Dokmak S, Ben Safta Y, Ftériche FS, Aussilhou B, Belghiti J. Pure laparoscopic right hepatectomy with the hanging maneuver for multiple hepatocellular adenomas. Ann Surg Oncol. 2014, in press. 